\title{
Tra metafisica e fisiologia. Il corpo quale fenomeno unitario in Schopenhauer, Cabanis e Bichat
}

\author{
Between metaphysics and physiology. Body as unitary phenomenon in \\ Schopenhauer, Cabanis and Bichat
}

Fiorella Giaculli'

\begin{abstract}
Il presente saggio si propone di analizzare un aspetto dell'interpretazione schopenhaueriana della corporeità: la natura unitaria del corpo umano. Per Schopenhauer il Leib, pur nella sua pluralità di significati, è riconducibile a un'unità di fondo, l'essere oggettivazione della volontà. Inoltre, il corpo inteso come organismo e come insieme di affezioni e di pensieri è concepito come unione di parti che si relazionano e si suggestionano reciprocamente. Per Schopenhauer, questa interpretazione è comprovata e condivisa, dal punto di vista fisiologico, da Cabanis e Bichat, nei termini di coappartenenza di physique e moral per l'uno, e di interconnessione tra la vie organique e la vie animale per l'altro. Il saggio si propone dunque di illustrare tale visione unitaria e di tratteggiare in che misura queste diverse prospettive si incontrano. Per descrivere tale interpretazione, il saggio si compone di tre paragrafi introduttivi, volti a inquadrare la questione centrale, trattata nei paragrafi quarto e quinto. Nel primo paragrafo è accennata la funzione centrale che il corpo umano svolge per la metafisica schopenhaueriana; nel secondo è tratteggiata l'importanza che la fisiologia riveste per il pensiero del filosofo; nel terzo è abbozzato l'incontro di Schopenhauer con i due fisiologi francesi, approfondito nei paragrafi successivi. Nel quarto paragrafo sono presentati i punti di contatto, e di divergenza, tra la posizione schopenhaueriana e cabanisiana, circa la natura monistica della corporeità. $\mathrm{Nel}$ quinto sono descritte le somiglianze tra il pensiero schopenhaueriano e bichatiano, intorno al corpo umano. Attraverso l'analisi dell'interpretazione schopenhaueriana, cabanisiana e bichatiana, della corporeità, emerge un'idea di Leib quale fenomeno unitario, che presenta elementi in comune tra la prospettiva filosofica e fisiologica.
\end{abstract}

Parole chiave: Corpo; Unità; Fisiologia; Schopenhauer; Cabanis; Bichat

\begin{abstract}
The following essayaims toanalyse one aspect of schopenhauerean interpretation of body: the idea of Leib such as unitary phenomenon. According to Schopenhauer, human body, although its polysemy, is ascribable to an underlying unity, the objectivation of the will. Besides, the body considered as organism and as combination of affections and thoughts is conceived as a unity of parts which relate and influence mutually. According to Schopenhauer, this interpretation is proved and shared, from a physiological point of view, by Cabanis and Bichat, in terms of belonging between physique and moral on the one hand, and in terms of interconnection between vie organique and vie animale on the other hand. The essay aims to describe this unitary interpretation and intends to illustrate to what extent the different perspectives agree. To describe this interpretation, the essay is made up of three introductive paragraphs, to contextualize the main question, analysed in the fourth and in the fifth paragraph. In the first paragraph is outlined the pivotal function that human body has for schopenhauerean metaphysics; in the second one is
\end{abstract}


sketched the relevance that physiology has for the philosopher's thought; in the third one it is traced Schopenhauer's reading of french physiologists, examined in depth in the following paragraphs. In the fourth paragraph are presented common points, and differences, between Schopenhauer and Cabanis' position, about monistic nature of body. In the fifth one, are described similarities between Schopenhauer and Bichat' thought, on corporeity. Through the analysis of Schopenhauer, Cabanis and Bichat' interpretation on corporeity, comes to light an idea of Leib, such as unitary phenomenon, which has common points between the philosophical and the physiological perspective.

Keywords: Leib; Unity; Physiology; Schopenhauer; Cabanis; Bichat

\section{II corpo umano tra polisemia e unità}

Nel $\S 18$ de Il mondo come volontà e rappresentazione, Schopenhauer presenta il corpo umano quale fenomeno in grado di disvelare la natura delle cose. Attraverso il suo agire, intenzionale e irriflesso, le sue affezioni, la sua struttura organica, il corpo umano suggerisce al filosofo che l'intero mondo corporeo è oggettivazione della volontà.

Il corpo è "visibilità" della volontà poiché ogni suo fare e non fare esprime deliberazione o non intenzionalità, volontà in una connotazione molto comune del termine:

L'atto di volontà e l'azione del corpo non sono due stati diversi oggettivamente conosciuti, che siano collegati dal vincolo della causalità, non stanno fra loro nel rapporto di causa ed effetto; ma sono una stessa e unica cosa [...]. L'azione del corpo non è nient'altro che l'atto di volontà oggettivato, ossia entrato nell'intuizione (W I, § 18, p. 223).

Il corpo è "specchio" della volontà anche nel significato di passione ${ }^{1}$. Il corpo è espressione del velle e del nolle nell'accezione del desiderare e aborrire, cercare e rifuggire, nel senso del sentimento e dell'emotività. Non soltanto ogni moto del volere si rivela nella corporeità, ma il Wille suggestiona il Leib, e viceversa, in quanto elementi che si coappartengono: "Con ciò, che il corpo è solo la visibilità della volontà, si spiega l'esatto collegamento tra la volontà e il corpo, si spiega perché ogni passione, ogni affetto, cioè ogni gagliardo volere e non volere scuota tanto e modifichi il corpo" (HN I, p. 140).

Il proprio corpo è dunque “immagine" della volontà nei significati di deliberazione, non intenzionalità e affezione. Primariamente, asserire che il corpo è manifestazione della volontà significa che esso è visibilità di una "spinta” verso la vita che desidera perdurare.

\footnotetext{
${ }_{1}$ Sulla valenza del Wille come passione, si consideri rappresentativo di tale significato un passo di Sulla libertà del volere umano: "Certo, per volere non si hanno da intendere soltanto i decisi atti di volontà che diventano subito azione e le risoluzioni formali accanto alle azioni che ne derivano; ma chi è in grado di afferrare l'essenziale anche in mezzo a diverse modificazioni di grado e di natura non esiterà ad annoverare tra le manifestazioni della volontà anche ogni brama, aspirazione, desiderio, struggimento, anelito, speranza, amore, gioia, giubilo e simili, e non meno ogni non volere, ogni riluttanza, ogni aborrire, fuggire, temere, essere in collera, odiare, affliggersi, soffrire, insomma tutte le passioni e i moti dell'animo, dato che queste passioni e questi moti sono soltanto più o meno deboli o forti, ora violenti e tempestosi, ora calmi e lievi moti della propria volontà o ostacolata o scatenata, soddisfatta o non soddisfatta; e tutti riguardano il raggiungimento o il fallimento del voluto, la sopportazione o il superamento di ciò che si aborrisce, in svariate forme: essi sono dunque decise affezioni di quella stessa volontà che si attiva nelle decisioni e nelle azioni. A questo campo appartengono perfino quelli che chiamiamo sentimenti di piacere e di dolore: essi esistono in una grande varietà di gradi e di specie, ma è sempre possibile farli risalire a moti di desiderio o di aborrimento, cioè alla volontà stessa soddisfatta o insoddisfatta, ostacolata o scatenata, che prende coscienza di sé, anzi ciò si estende fino alle sensazioni del corpo, piacevoli o dolorose, e a tutte le infinite sensazioni che stanno fra questi due estremi, dato che la natura di tutti questi moti dell'animo consiste nell'entrare direttamente nell'autocoscienza come conformi o contrari alla volontà" (E I, pp. 51-52).
} 
Come la singola azione del corpo oggettivizza il singolo atto della volontà, così tutto il corpo, nel suo esserci, deve oggettivare l'intera natura del volere, l'intera volontà, il carattere intelligibile. Dunque, l'intero corpo deve trovarsi con la mia volontà in generale, nella stessa relazione in cui ogni singola azione del corpo si trova rispetto al singolo atto volitivo che essa rappresenta (V II, p. 35).

La volontà si oggettiva originariamente nel sangue, "liquido primordiale" il cui moto perenne riflette il tendere inesausto del volere. Esso crea, plasma e nutre l'organismo; forma dapprima i suoi stessi vasi, poi i muscoli, e tutt'uno con esso è il cuore, primum vivens et ultimum moriens. La volontà si esprime in ogni apparato, in ogni parte del corpo, immagine delle "tendenze fondamentali" dell'impulso vitale. Si esprime negli organi di senso con diverse sfumature. Infine, si oggettiva come sistema nervoso. Per Schopenhauer finanche il volto esprime la volontà, la volontà individuale, il carattere.

L'esperienza del proprio corpo rappresenta dunque la chiave di volta della metafisica schopenhaueriana, giacché "passaggio sotterraneo" in grado di approssimarsi al fondamento che custodisce e incarna; fondamento comune all'intero mondo corporeo.

\section{Schopenhauer e la fisiologia}

Nella misura in cui il corpo umano rivela il principio imperituro, non dovrebbe stupire che Schopenhauer si sia dedicato alla fisiologia; del resto, il filosofo si è sempre interessato agli studi scientifici, è quindi una scelta in linea con la sua formazione.

L'attenzione rivolta alla fisiologia non subentra a partire dall'elaborazione della metafisica della volontà, ma la precede, risalendo al periodo universitario; inoltre, sarebbe rimasta costante nel tempo: si pensi agli esperimenti personalmente condotti sulla fisiologia della vista, agli aggiornamenti attraverso le letture dell'Archiv für die Physiologie, e agli studi delle ricerche in materia.

Ora la fisiologia è funzionale a un aspetto della filosofia, come la metafisica e la gnoseologia, ora è declinata in relazione all'ottica, ora all'anatomia. La fisiologia non si configura però come traduzione dell'argomentazione filosofica; né, viceversa, la filosofia è traduzione dell'analisi fisiologica. I due saperi si confrontano, all'occorrenza si mettono in discussione reciprocamente, si completano ${ }^{2}$.

Da studente, Schopenhauer segue i corsi di fisiologia di Blumenbach a Gottinga; frequenta le lezioni Horkel a Berlino, attraverso cui conosce il ruolo che Reil, fondatore del summenzionato Archiv, ha per lo sviluppo della nuova fisiologia. Horkel, come Reil, è dedito alla ricerca scientifica, ed è fautore della fisiologia quale disciplina fondante la conoscenza dei viventi. "Dalla sua voce Schopenhauer poté nuovamente avvicinare, dopo le fondamentali lezioni di Blumenbach, alcune questioni rilevanti della fisiologia, quali il rapporto con la Naturphilosophie (con il riferimento a un libro di Karl Eberhard Schelling) e con la frenologia di Gall ${ }^{3}$.' Gall e Reil sono figure di riferimento anche dei corsi di Rosenthal, sull'anatomia del cervello e sulla fisiologia del sistema nervoso, che Schopenhauer segue a Berlino. Anche le letture della Critica del giudizio e degli scritti

Circail ruolo della fisiologia per la filosofia schopenhaueriana, si considerino, traglialtri, iseguentistudi: JANET, Schopenhauer et la physiologie française. Cabanis et Bichat, pp. 35-59; KLOPPE, Die Bestätigung der Philosophie Arthur Schopenhauers (17881860) durch das Werk des Physiologen Xavier Bichat (1771-1802), pp. 306-312; LEFRANC, Schopenhauer lecteur de Cabanis, pp. 549-557; MANDELBAUM, The Physiological Orientation of Schopenhauer's Epistemology, in FOX (Hrsg.), Schopenhauer. His Philosophical Achievement, pp. 50-67; SCHMIDT, Physiologie und Transzendentalphilosophie bei Schopenhauer, pp. 4353; SEGALA, Fisiologia e Metafisica in Schopenhauer, pp. 35-66; SEGALA, Schopenhauer, la filosofia, le scienze, pp. 177-357; SEGALA, The Role of Physiology in Schopenhauer's Metaphysics of Nature, pp. 327-334; TAKAHASHI, Lebensphänomenologie durch Physiologie in der mittleren und späten Periode Schopenhauers, pp. 163-174.

SEGALA, Schopenhauer, la filosofia, le scienze, p. 237. 
schellinghiani sulla filosofia della natura sono fondamentali per la rilevanza della fisiologia nell'indagine sul vivente. Probabilmente, a partire dalla lettura di Schelling, Schopenhauer scopre e studia Kielmeyer, in particolare lo scritto Über die Verhältnisse der organischen Kräfte .

Terminati gli studi, grazie all'incontro con Goethe e al suo invito a occuparsi della propria Teoria dei colori, Schopenhauer si cimenta nella sperimentazione ottica e cromatologica e nel 1816 pubblica Sulla vista e $i$ colori ${ }^{5}$. Si tratta di una ricerca che riceverà diversi riconoscimenti, ad esempio da parte dell'Accademia delle Scienze di Monaco; di Justius Radius, che inserisce lo scritto schopenhaueriano, tradotto in latino, nella collana Scriptores ophthalmolgici minores; del premio Nobel per la chimica Wilhelm Ostwald, che scorge nell'ipotesi della percezione del colore, mediante la divisione qualitativa dell'attività della retina, un'anticipazione delle teorie cromatiche di Hering ${ }^{6}$. Al di là dei riconoscimenti, è uno scritto significativo poiché mostra la propensione di Schopenhauer a far dialogare la filosofia con altri saperi e l'adoperarsi personalmente nella sperimentazione.

Schopenhauer è lettore della Fisiologia di Burdach, degli studi di Haller e di Cuvier, per limitarsi ad alcuni non sporadici riferimenti da parte del filosofo.

Egli dedica inoltre due capitoli significativi alla fisiologia, Fisiologia e patologia e Fisiologia vegetale, in Sulla volontà nella natura, scelta che indica la centralità della fisiologia per la filosofia della natura e dunque per il confronto tra la "scienza del particolare", la fisica, e la "scienza dell'universale", la metafisica.

\section{Schopenhauer lettore dei Rapports du physique et du moral de l'homme di Cabanis e delle Recherches physiologiques sur la vie et la mort di Bichat}

Tra gli anni venti e quaranta Schopenhauer scopre due fisiologi francesi, Pierre Jean Georges Cabanis e Marie François Xavier Bichat, che a suo parere supportano dal punto di vista fisiologico alcuni aspetti della sua filosofia. Cabanis sarebbe stato scoperto intorno al 1825 e Bichat intorno al 1838, date che indicano che la metafisica schopenhaueriana non si è modellata sulla loro fisiologia, ma è sorta indipendentemente, per incontrarsi solo poi con essa ${ }^{7}$.

Schopenhauer osserva che l'unità tra il Leib e l'objectiviert Wille è corroborata dal punto di vista fisiologico dai due francesi. È espressa da Cabanis nei termini di rapports du physique et du moral e da Bichat nei termini di relazione tra la vie organique e la vie animale.

\footnotetext{
${ }^{4}$ A riguardo, si veda SEGALA, Fisiologia e metafisica in Schopenhauer, pp. 53-66.

${ }^{5}$ Circa l'incontro tra Schopenhauer e Goethe, il convergere e il divergere in merito alla Farbenlehre, si legga, tra gli altri, GRIGENTI, Goethe e Schopenhauer: la natura dei colori e i colori della natura, in GRIGENTI, Natura e rappresentazione. Genesi e struttura della natura in Arthur Schopenhauer, pp. 117-212; SCHUBBE, SØREN, Schopenhauer und Goethe. Biographische und philosophische Perspektiven; SEGALA, Schopenhauer, Goethe el'ottica sperimentale, pp. 217-231; si consideri anche il Carteggio con Goethe, in SCHOPENHAUER, La vista e i colori e carteggio con Goethe, pp. 115-147.

6 Sulla fortuna della monografia sui colori, cfr. ad esempio VOLPICELLI, A. Schopenhauer, La natura vivente ele sue forme, pp. 22-23.

7 In merito al periodo della lettura dei due fisiologi, così precisa Segala: "Schopenhauer venne infatti a conoscenza dell'opera bichatiana soltanto una ventina di anni dopo la stesura della prima edizione del Mondo come volontà e rappresentazione. Hübscher ritiene che soltanto nel 1838 la lettura delle Recherches sia stata completata, e il fatto che ne La volontà della natura non vi sia alcun riferimento ad esse fa propendere per il periodo compreso tra il 1835 e il 1838. È vero che nei manoscritti dedicati alle riflessioni sul primato della volontà sull'intelletto, redatti per più di un decennio a partire dal 1826, si trova spesso il nome di Bichat, ma le differenze calligrafiche indicano che si tratta di aggiunte successive. Ciò significa che soltanto dopo la lettura delle Recherches physiologiques sur la vie et la mort Schopenhauer si rese conto della convergenza tra le proprie idee e quelle dello scienziato francese. Quindi è corretto sostenere che Schopenhauer impostò la propria concezione della fisiologia senza essere guidato dalle idee di Bichat. [...] Schopenhauer menzionò per la prima volta i Rapports in una corposa annotazione risalente alla fine del 1825 e li definì un gehaltvolles Buch. Ciò chiarisce che l'attribuzione di un ruolo essenziale alla fisiologia all'interno della filosofia della natura del Mondo come volontà e rappresentazione fu indipendente anche da Cabanis". SEGALA, Fisiologia e metafisica in Schopenhauer, pp. 50-51.

\& Sul concetto di riprova, si considerino in particolare la Prefazione e l'Introduzione a Sulla volontà nella natura, come del resto l'opera stessa.
} 
Tale visione comporta il superamento di un certo dualismo a favore di un'interpretazione monistica della corporeità ${ }^{9}$ : organismo, pensieri e passioni non sono intesi come entità contrapposte, l'una sensibile, l'altra metasensibile, ma sono concepiti come aspetti del fenomeno composito che il corpo è ${ }^{10}$. Chiaramente, per Schopenhauer sussiste un elemento metafisico che i due fisiologi escludono; ma, nella misura in cui tale elemento si manifesta come corpo, allora ogni sua aspetto è corporeo. Per Schopenhauer, "tutti i fenomeni, anche quelli mentali, sono fisici", e, nel contempo, "ogni cosa fisica è d'altra parte insieme una cosa metafisica” (W II, Kap. 17, pp. 1337, 1339).

Muovendo da tale concezione unitaria, la questione su cui si intende soffermarsi concerne la fisiologia (e filosofia) delle affezioni, la quale rivela che l'elemento corporeo e l'elemento intellettivo ed emotivo non sono scissi. Non solo sono parti di un'unica realtà, ma si suggestionano mutuamente.

\section{Leib e Wille, physique e moral}

Diverse sono le circostanze in cui Schopenhauer menziona Cabanis, e tuttavia riconducibili a una stessa questione. Il filosofo tedesco e il fisiologo francese condividono una visione unitaria secondo la quale l'anima, un'accezione della volontà fenomenica in termini schopenhaueriani e il morale in quelli cabanisiani, è una parte del corpo: è quell'insieme di sentimenti, pensieri, abiti, non disgiunto dall'organismo, che si esprime infatti come corpo.

Il pensare è concepito da entrambi come una funzione corporea, che abbisogna di riposo al pari delle altre funzioni, che si affatica se le membra sono stanche, che è condizionato dalle emozioni e che a sua volta le condiziona.

Con parole schopenhaueriane,

Si deve in genere comprendere bene che il nostro pensiero non è altro se non la funzione organica del cervello, e si comporta quindi analogamente, per quanto riguarda il lavoro e il riposo, a ogni altra attività organica. Uno sforzo eccessivo, come rovina gli occhi, così fa del cervello. Si è detto a ragione: il cervello pensa come lo stomaco digerisce. L'illusione di un'anima immateriale, semplice e pensante essenzialmente e continuamente, di conseguenza instancabile, che nel cervello abita soltanto, e non ha bisogno assolutamente di nulla a questo mondo, ha di certo indotto molte persone a comportamenti assurdi e all'intorpidimento delle loro forze spirituali. [...] Ci si deve abituare a vedere le proprie forze intellettuali come delle funzioni del tutto fisiologiche, trattandole del tutto di conseguenza, cioè

Questo elemento è sottolineato da Segala, il quale aggiunge che l'interpretazione cabanisiana e bichatiana era per Schopenhauer ancora più ammirevole poiché non pretendeva di sostituirsi a una spiegazione filosofica della realtà: "Quando parlava di costoro [Ludwig Büchner e Jacob Moleschott], Schopenhauer non nascondeva il proprio fastidio per un materialismo rozzo che si arrogava il diritto di ergersi a sistema e di sostituirsi alla metafisica. Al contrario, I'analisi del rapporto tra il fisico e il morale nelle opere di Bichat e Cabanis costituiva un nuovo e sicuro argine contro il dualismo cartesiano e lo spiritualismo, ma non una resa al riduzionismo e al materialismo assurti a sistema. II merito dei due grandi fisiologi francesi era quello di avere sviluppato una concezione scientifica di prim'ordine che non aveva l'ambizione di sostituirsi alla metafisica, ma che era stata in grado di porre le basi per una positiva interazione con la metafisica". SEGALA, Fisiologia e metafisica in Schopenhauer, p. 46. Secondo Lefranc, al superamento del dualismo subentra la diade Wille-Vortstellung: "Mais Schopenhauer ne lit pas seulement dans Cabanis ou Bichat une critique radicale du dualisme de l'âme e du corps. Il y trouve, déjà nettement indiqué, un nouveau idealisme de l'intellect et de la volonté qui constitue une révolution philosophique essentielle et qui n'est rien d'autre que l'afflleurement de la distinction du phénomène et de l'en-soi". LEFRANC, Schopenhauer lecteur de Cabanis, p. 554.

${ }^{10}$ A partire dall'importanza che sia Cabanis, sia Bichat attribuiscono alla conoscenza del corpo, per la comprensione dell'uomo, segue un'idea di "science de l'homme" che conferisce alla medicina un ruolo nodale e che riconsidera gli altri saperi e i loro rapporti. Nel discorso preliminare di Bichat, al Premier Recueil de la Société médicale d'émulation, è evidente l'intento di riconoscere alla medicina una funzione cen 
risparmiandole, sforzandole, eccetera, e a pensare, che ogni sofferenza fisica, ogni molestia, ogni disordine, in qualunque parte si verifichi, impressiona la mente. A seguire questa norma, più di ogni altro insegna Cabanis, in Des rapports du physique et du moral de l'homme (P I, p. 599).

L'irriducibilità dell'organismo o il suo alludere a qualcosa d'altro segnano la differenza tra i due pensatori: per Schopenhauer sussiste un principio che soggiace al morire dei corpi. In tal senso, è presente un elemento metafisico che, pur rivelandosi nel corpo, non coincide con esso. Al contrario, per Cabanis il corpo non rimanda a nient'altro da sé. Se anche suggerisce principi vitali, esso evoca qualcosa di vago e incerto, impossibile da conoscere.

Questa diversità è ben compresa da Schopenhauer, che, nell'esprimere la sua ammirazione per gli studi cabanisiani, pone anche una distinzione tra la sua prospettiva e quella del fisiologo:

La vera fisiologia, al suo più alto livello, mostra che ciò che nell'uomo è spirituale (la conoscenza) è un prodotto del suo fisico, e ciò ha fatto, come nessun altro, Cabanis; ma la vera metafisica ci insegna che questo stesso fisico è mero prodotto, o piuttosto fenomeno di un che di spirituale (la volontà), anzi che la materia stessa è condizionata dalla rappresentazione, nella quale soltanto esiste. L'intuizione e il pensiero vengono sempre più spiegati in base all'organismo, ma non il volere, bensì al contrario in base a questo è spiegato l'organismo $(\mathrm{N}, \mathrm{p} .78)$.

Per Schopenhauer non solo il corpo umano, ma l'intera Körperwelt è l'unica realtà che si può, e si deve, indagare e interpretare; tuttavia essa rimanda a ciò che, pur rivelandosi nel fenomeno, non si risolve in esso ed è indipendente dalle sue leggi. La corporeità è il "rivestimento più tenue" che esprime qualcosa d'altro, non soggetta al tempo, allo spazio, alla causalità. Il corpo è il fenomenizzarsi di questo altro, che in esso si rivela relativamente, ossia in relazione a qualcosa, e non in assoluto, per sé. Il corpo, con la sua struttura, le sue azioni, i suoi pensieri, le sue passioni, è pertanto fisico e metafisico insieme, nella misura in cui è concrezione del tendere senza posa, che aspira a conservarsi, ed è caduco come tutte le naturae res, soggetto alle loro leggi.

C'è unità tra un pensiero, un desiderio, un sentimento e l'organismo; ma il morale o il volere (termini sostanzialmente sinonimici, nelle accezioni a cui ci stiamo riferendo in questo contesto) seppur non altri dalla corporeità, per Schopenhauer non possono essere ricondotti soltanto al fisico, poiché quest'ultimo non si spiega esclusivamente da sé, ma deve essere posto in relazione a quella tensione originaria, metafisica, che è l'aspirazione alla vita, di cui è fenomeno. Per Schopenhauer dunque la redutio ad unum è di natura

trale, senza tuttavia trascurare lo studio di altre discipline, che concorrono alla comprensione dell'uomo: "Appassionati della nostra Arte, avidi di nuove conoscenze, vorremmo costringere tutte le scienze umane a pagare un giusto tributo alla Medicina; così amiamo le Lettere poiché esse possono gettare fiori su una scienza sublime e bella. [...] Amiamo le Scienze matematiche perché esse formano lo spirito di metodo e d'analisi [...], amiamo la Morale poiché senza di essa si ha dell'Uomo solo una conoscenza imperfetta, grossolana e materiale; amiamo la Fisica perché noi stessi siamo un elemento del grande sistema del mondo; amiamo la Chimica poiché obbliga la Natura a rivelarci i suoi segreti e i suoi profondi misteri; amiamo la Storia naturale, con una parola, amiamo la filosofia universale poiché siamo convinti che una teoria medica sarà più saggia e meglio costituita se più strettamente si identificherà con la scienza generale di cui la medicina pratica è solo un corollario o una applicazione. [...] Tuttavia, studiandole tutte, cercheremo di sottrarci a eccessi funesti”. Se Bichat sottolinea il ruolo imprescindibile della medicina, da porsi in relazione a molte altre branche del sapere, Cabanis considera la medicina e la morale quasi come il recto e il verso di una stessa indagine. Scrive infatti in Coup d'œil sur les révolutions et sur la réforme de la médecine: "Si comincia a riconoscere oggigiorno, che la medicina e la morale sono due branche della stessa scienza, che, riunite, formano la scienza dell'uomo. L'una e l'altra riposano su di una base comune, sulla conoscenza fisica della natura umana. Ė infatti attraverso la fisiologia che esse devono ricercare la soluzione concernente tutti i loro problemi, il punto di appoggio di tutte le verità speculative e pratiche. Dalla sensibilità fisica, o dall'organizzazione che la determina e la modifica, derivano in effetti le idee, i sentimenti, le passioni, le virtù ed i vizi". Ebbene, la considerazione del corpo quale unica realtà e quale fenomeno unitario conduce a un diverso metodo di ricerca e a una diversa antropologia. 
metafisica; per Cabanis è invece fisica. Per Schopenhauer l'unità è data da qualcosa di diverso dal corpo, che nondimeno in esso soltanto si esprime. L'unità si innesta su un principio che non si esaurisce nella körperlichkeit, ma che soggiace a essa, rendendola possibile e rendendosi così visibile; per Cabanis ciò che una certa tradizione voleva duale è unità essenzialmente organica, composta di fisico e morale ${ }^{11}$.

Inoltre, che per il fisiologo francese lo studio dell'uomo, e dal punto di vista medico e dal punto di vista filosofico, debba essere indagine intorno all'organismo, è chiaro sin dall'Introduzione ai Rapporti:

Lo studio dell'uomo fisico è altrettanto interessante per il medico e per il moralista: esso è quasi necessario ad entrambi. Sforzandosi di scoprire i segreti dell'organismo, osservando i fenomeni vitali, il medico cerca di individuare in che cosa consiste lo stato di perfetta salute, [...]. Il moralista si sforza di risalire fino alle operazioni più oscure che costituiscono le funzioni dell'intelligenza e le determinazioni della volontà. [...] Gli scrittori che si sono occupati in modo abbastanza approfondito dell'analisi delle idee, di quella del linguaggio o degli altri segni che le rappresentano e dei principi della morale privata o pubblica, hanno quasi tutti avvertito la necessità di farsi guidare nelle loro ricerche dalla conoscenza della natura umana fisica. Come è possibile, infatti, descrivere con esattezza, valutare e delimitare senza errore i movimenti di una macchina e i risultati della sua azione se non si conoscono prima la sua struttura e le sue proprietà? [...] Ogni filosofo ha elaborato la propria teoria dell'uomo. Coloro stessi i quali, per spiegarne le vari funzioni, hanno creduto di dover supporre in esso due forze di natura diversa, hanno anch'essi riconosciuto che è impossibile sottrarre le operazioni intellettuali e morali al potere del fisico $^{12}$.

Cerchiamo ora di esplicare in che termini il fisiologo descrive la relazione tra le parti dell'unita corporea, e di comprendere in che misura si possono ravvisare punti di contatto con la metafisica schopenhaueriana.

L'analisi cabanisiana a cui il filosofo fa riferimento è esposta, in particolare, nei Rapports du physique et du moral de l'homme. L'opera si compone di dodici Memorie, lette diacronicamente all'Istituto nazionale per le scienze e le arti, ed edite nell' $1802^{13}$, che si propongono di illustrare quanto annuncia già il titolo: la relazione tra il fisico e il morale nell'uomo, intendendo con il primo termine l'organismo e con il secondo l'insieme di idee, volizioni, comportamenti. Si tratta di espressioni che non indicano due entità opposte, ma aspetti diversi di una realtà corporea unitaria. I loro rapporti riguardano quindi i modi

\footnotetext{
${ }^{11}$ Occorre precisare che Cabanis non esclude toto coelo l'esistenza di principi vitali, piuttosto li considera inconoscibili mediante gli strumenti che l'uomo possiede. Nell'Introduzione ai Rapports, egli è chiaro a riguardo: "Alcuni individui sono parsi credere, a quanto mi si dice, che quest'opera aveva come scopo, o come conseguenza, quella di rovesciare talune dottrine e di stabilirne delle altre relativamente alla natura delle cause prime. Ma questo non può essere vero: e con un po' di riflessione e di buona fede non è neppure possibile crederlo seriamente. Nel corso dell'opera il lettore vedrà spesso che noi consideriamo queste cause situate fuori dalla sfera delle nostre ricerche, e come sottratte, e per sempre, ai mezzi di investigazione che l'uomo ha ricevuto insieme al soffio vitale. [...] Lasceremo dunque a spiriti più fiduciosi, o se si vuole più illuminati, la cura di ricercare, attraverso strade che consideriamo impraticabili per noi, qual è la natura del principio che anima i corpi viventi". CABANIS, Rapporti tra il fisico e il morale dell'uomo, traduzione parziale, pp. 17-18. Si può considerare Schopenhauer uno "spirito più fiducioso", che ha scorto il "soffio vitale" non in una strada altra rispetto a quella percorsa da Cabanis, ma nella stessa: il corpo umano.

12 Ibidem, pp. 3-4.

${ }^{13}$ Le Memorie sono le seguenti: I. Considerazioni generali sopra lo studio dell'uomo, e sopra i rapporti della sua organizzazione fisica con le sue facoltà; II. Storia fisiologica delle sensazioni; III. Continuazione della storia fisiologica delle sensazioni; IV. Dell'influenza delle età sulle idee e sulle affezioni morali; V. Dell'influenza dei sessi sul carattere delle idee e delle affezioni morali; VI. Dell'influenza de' temperamenti sulla formazione delle idee e delle affezioni morali; VII. Dell'influenza delle malattie sulla formazione delle idee e delle affezioni morali; VIII. Dell'influenza del regime sulle disposizioni e abitudini morali; IX. Dell'influenza dei climi sopra le abitudini morali; X. Considerazioni relative alla vita animale; XI. Dell'influenza del morale sul fisico; XII. Dei temperamenti acquisiti.
} 
del coesistere di elementi distinti dello stesso fenomeno ${ }^{14}$. Come le parti del physique sono relate le une alle altre, influenzandosi a vicenda, così le parti del moral. Inoltre, fisico e morale intessono mutue relazioni. Uno stato d'animo si traduce in un'espressione del volto, in un atto del corpo, una sensazione gradevole o spiacevole si manifesta come umore. Tale esprimersi non è riduzione, non è coincidenza, ma coappartenenza tra le diverse componenti dell'esser corpo.

Nell'undicesima Memoria dei Rapporti, Dell'influenza del morale sul fisico Cabanis dà inizio alla sua riflessione muovendo dall'armonia cosmica a quella organica. L'universo è costituito di parti molteplici che si coordinano e si necessitano scambievolmente; di parti che compongono un ordine, necessario alla conservazione. Similmente, anche nell'organismo umano l'armonia è necessaria, affinché ciascuna parte possa relazionarsi alle altre senza alterare l'equilibrio complessivo e affinché il tutto possa mantenersi.

L'insieme di funzioni, apparati, organi, definibile con il termine fisico, è un continuum di azioni e interazioni tra le sue parti. Esso si rapporta anche al morale, insieme di pensieri, sentimenti, costumi, diverso dal primo, ma non totalmente altro. Anche 1'“organo del pensiero" partecipa dei rapporti organici:

[...] noi vediamo gli organi partecipare delle affezioni gli uni degli altri, entrare in movimento di concerto, eccitarsi scambievolmente, o bilanciarsi e contrariarsi nelle loro funzioni rispettive. Un legame comune li unisce; essi fanno parte del medesimo sistema. Il grado della loro sensibilità, la natura e l'importanza delle loro funzioni, taluni rapporti di situazione, di struttura, di scopo o di uso, determinano il carattere e fissano i limiti di questa influenza reciproca. [...] Or, le leggi che regolano, per esempio, tutti i visceri addominali, loro sono evidentemente comuni cogli organi del pensiero; questi ultimi vi sono evidentemente sottoposti senza alcuna restrizione. Se il sistema della vena-porta influisce sul fegato e la milza, la milza ed il fegato sullo stomaco, lo stomaco sugli organi della generazione, gli organi della generazione su gli uni e gli altri, e reciprocamente; l'organo cerebrale, considerato come quello del pensiero, e per lo stato abituale o passeggero che risulta per lui da questa funzione, non è ligato mercè rapporti meno stretti dell'influenza reciproca, col fegato, colla milza, collo stomaco, e colle parti della generazione ${ }^{15}$.

Asserire che anche il cervello si relaziona con gli altri organi, non soltanto perché guida i loro funzionamenti, ma anche perché è suggestionato da questi, significa ammettere la sua non indipendenza dall'organismo. Significa che una sua funzione peculiare, il pensare, non è slegata dalla realtà organica. Questo non implica che il pensiero sia tutt'uno con un certo funzionamento, o mal funzionamento corporeo, sarebbe oltre modo riduttivo, ma vuol dire che in quanto è elemento della corporeità, si sviluppa con essa, la influenza e ne vive gli influssi. Non solo il pensiero, ma anche il sentimento condiziona il corpo, e il pensiero, e ne è da questi condizionato. Per Cabanis si tratta di "un fatto generale incontrastabile", che può essere appurato dai moralisti e fisiologi, sino agli uomini più "grossolani”:

È un fatto, che secondo lo stato dello spirito, secondo la differente natura delle idee e delle affezioni morali, l'azione degli organi può essere alternativamente eccitata, sospesa, o totalmente invertita ${ }^{16}$.

\footnotetext{
${ }_{14}$ Puntualizza a riguardo Moravia: "Ben lungi dall'essere una polarità qualitativamente (o addirittura ontologicamente) diversa dal 'fisico', il 'morale' viene anzi presentato più d'una volta come un mero aspetto o parte dell'organismo materiale-unitario dell'uomo. [...] II 'morale', insomma, sembra identificarsi con la produzione di idee, affetti e volizioni da parte del 'fisico' - al massimo di una parte specifica del fisico". MORAVIA, Introduzione, in CABANIS, Rapporti tra il fisico e il morale dell'uomo, traduzione parziale, pp. XIX-XX.

${ }^{15}$ CABANIS, Rapporti tra il fisico e il morale dell'uomo, traduzione integrale, p. 253.

16 Ibidem, p. 254.
} 
Cabanis illustra tale correlazione tra le parti con molteplici descrizioni. Tra queste, il fisiologo presenta i rapporti tra la nutrizione e uno stato d'animo; l'apparato riproduttivo e una passione; il timore e il suo esprimersi come corpo:

\begin{abstract}
Un uomo vigoroso e sano ha fatto un buon pranzo: in mezzo a quel sentimento di benessere che spande allora in tutta la macchina, la presenza degli alimenti nello stomaco, la di loro digestione si esiegue con energia; ed i succhi digestivi li disciolgono con faciltà e rapidità. Quest'uomo riceve egli una cattiva nuova? O passioni tristi o funeste vengono ad elevarsi istantaneamente nel suo seno? Subito il suo stomaco ed i suoi intestini cessano d'agire sugli alimenti che essi racchiudono.

[...] Si sa che non esiste organo alcuno più sottoposto al potere dell'immaginazione, quanto gli organi della generazione. L'idea di un oggetto amabile li eccita piacevolmente; un immagine disgustosa li agghiaccia. La passione può quasi sempre accrescere di molto la potenza fisica dell'autore, anche negli individui più deboli, intanto il suo eccesso può anche talvolta, come lo aveva osservato Montaigne, distruggerla o paralizzarla momentaneamente presso gli uomini anche i più forti.

[...] Il timore abbatte e può annientare le forze muscolari e motrici: la gioia, la speranza, i sentimenti coraggiosi ne decuplano gli effetti: la collera può accrescerli in un certo modo indefinitamente ${ }^{17}$.
\end{abstract}

Anche quanto concerne la sfera intellettiva, da un giudizio a un ricordo alla comprensione stessa, è intimamente legato all'elemento emotivo, a tal punto da poter essere anche stravolto da questo:

Ma l'azione istessa della sensibilità non è men sottoposta all'impero delle idee e delle affezioni dell'animo. Sopra un uomo attristato da idee affannose, agitato da sentimenti crudeli, gli oggetti esterni producono altre impressioni, che se il medesimo uomo fosse dolcemente occupato d'immagini piacevoli, ed il suo animo in istato di soddisfazione e di riposo. Le impressioni sono in noi stessi, e non negli oggetti.

Questi non ne possono essere se non le occasioni. La maniera di sentire la loro presenza e la loro azione dipende soprattutto da ciò cui si è disposto: la volontà può anche talvolta snaturare interamente gli effetti che essi producono sull'organo sensiente ${ }^{18}$.

Anche Schopenhauer osserva la profonda correlazione tra un'affezione e la corporeità: si arrossisce per l'imbarazzo, si impallidisce per lo spavento, si piange per la disperazione e per la commozione, si prova un senso di nausea alla vista di cose disgustose, il battito cardiaco si accelera per la rabbia e per la contentezza. "Inoltre un continuo dispiacere mina in profondità l'organismo, e lo spavento, come pure la gioia improvvisa, possono avere conseguenze mortali” (N, p. 88). In quanto fautore di tale monismo, Schopenhauer non poteva che trovare in Cabanis punti di contatto con il suo pensiero, e dunque conferme fisiologiche alla sua filosofia.

L'altro aspetto che emerge insieme al carattere unitario del corpo è che la sua natura è in primo luogo senziente ${ }^{19}$. È un tratto ulteriore che accomuna i due pensatori,

\footnotetext{
17 Ibidem, pp. 254-255.

18 Ibidem, p. 255. Secondo Cabanis, non soltanto uno stato d'animo, ma anche un funzionamento organico può influenzare un pensiero, ciò a ribadire la reciprocità degli influssi tra le parti del corpo. Emblematico a riguardo è il caso della nutrizione: "Pienezza o vacuità, attività o inerzia, ben-essere o mal-essere dello stomaco, tutto in una parola, fino alle singolarità più fuggitive del suo gusto e dei suoi appetiti, va a risonare all'istante nel centro cerebrale: e spesso si ritrovano le tracce di questi minori capricci nel carattere o nella conformazione delle idee, e nelle determinazioni volontarie le più distinte, del pari che nelle inclinazioni istintive le meno ragionate". Ibidem, p. 263.

19 Si tratta di un'attribuzione significativa, propria di un'antropologia in contrasto con quella che ha considerato l'uomo composto di due nature antitetiche, l'una materiale, l'altra spirituale; conferendo le funzioni complesse e superiori soltanto a quest'ultima, ritenendo le funzioni intellettive e affettive come indipendenti dall'organismo. Come sintesi di tale questione,
} 
poiché per Schopenhauer l'essenza umana è volente. Tale caratteristica non implica punto un misconoscimento logico, ossia del logos, ma un suo ridimensionamento: il filosofo attribuisce alla ratio una posizione secondaria, in termini temporali e di necessità in riferimento alla natura tutta, ma è ben lungi dal mettere in dubbio la sua rilevanza, essendo peraltro prodotto della voluntas funzionale alla vita, e strumento che consente all'uomo di pervenire alla volontà mediante l'autocoscienza. Per Cabanis, il sistema nervoso è un organo guida fondamentale, senza il quale non si avrebbero animazione, sensibilità, coordinazione, ricezione e reazione. Nondimeno, non può considerarsi il fulcro dell'essenza umana, né è l'unica guida dell'organismo: esistono altre guide decentrate e distribuite in tutto il corpo. Inoltre, l'influenza che il sistema nervoso esercita non lo esime dall'essere influenzato a sua volta.

La catena ininterrotta di relazioni, funzioni, movimenti, lega tutte le parti del corpo:

[...] ciascuna [funzione] è ligata da tutte le altre, per mezzo di relazioni più o meno dirette; che esse devono eccitarsi e sostenersi scambievolmente; che conseguentemente esse formano un cerchio nel quale gira la vita, mantenuta da questa reciprocità d'influenza ${ }^{20}$.

\section{Wille, Vie organique e vie animale}

Se i rimandi a Cabanis concernono essenzialmente l'unità tra il sentire, il pensare e la corporeità, i riferimenti a Bichat hanno un carattere più esteso, riguardante non soltanto la natura unitaria del corpo, ma anche l'intera analisi che il médecin-philosophe tratteggia della vita e della morte. Schopenhauer osserva con stupore il reciproco completarsi tra la propria ricerca e quella bichatiana, fornendo punti di vista diversi, ma complementari, rispetto al comune oggetto d'indagine:

Le sue considerazioni e le mie si sostengono vicendevolmente, fornendo le sue il commento fisiologico delle mie, e queste il commento filosofico delle sue; e ci si comprenderà nel modo migliore leggendoci insieme da entrambe le parti. [...] Egli pone a base delle sue argomentazioni la contrapposizione fra vita organica e vita animale, che corrisponde alla mia fra volontà e intelletto. Chi bada al senso e non alle parole, non si farà trarre in inganno

del cambiamento inaugurato da Cabanis, si consideri una riflessione di Moravia: "Un indirizzo centrale della tradizione d'Occidente aveva identificato - lo sappiamo bene - il nucleo centrale di tale essere con l'esprit o con il logos, col pensiero o con la ragione. Ma tutto ciò appare ora, se non errato, almeno unilaterale. L'uomo è anzitutto vita; e la vita è anzitutto sensibilità: la sensibilità - si legge nei Rapporti - è il «fatto generale» della natura vivente. Bisogna dunque ridimensionare anche lo stesso pensiero, lo stesso cogito. Al principio ben noto di Cartesio «cogito ergo sum» verrebbe quasi da sostituire il principio «sentio ergo sum». In effetti, per Cabanis, è «dal momento che sentiamo [che] siamo». Questa tesi, questa presa di posizione hanno un peso di valore davvero storico. [...] Non già, si badi, che Cabanis intenda svalutare la dimensione intellettuale dell'essere umano, ma è indubbio ch'egli attribuisce un rilievo assolutamente inconsueto e tendenzialmente privilegiato a una dimensione 'altra' di tale essere. L'uomo non è più essenzialmente o primariamente raziocinio, ma è sensibilità. Intrecciate alle idee, anzi rispetto ad esse per più versi precedenti e più caratterizzanti, vi sono le sensazioni, i sentimenti, le passioni. Analizzare l'essere umano implica, prima di tutto, cogliere questa dimensione del 'sentire': una dimensione, è da sottolineare, che ha in Cabanis una valenza non meramente gnoseologica (il 'sentire' come componente più o meno subalterna del 'conoscere') ma più ampiamente antropologica. L'uomo come essere costitutivamente sensibile, come être de désir, come soggetto passionale - quell'uomo che di lì a non molto diverrà il protagonista di un certo versante del Romanticismo europeo - trova nei Rapports du physique et du moral de l'homme un punto di riferimento ineludibile. A questo proposito è ben noto quanto due dei massimi teorici ottocenteschi dell'essere umano come essere senziente/desiderante Stendhal e Schopenhauer - abbiano conosciuto, letto e apprezzato l'opera cabanisiana (e l'opera degli idéologues in genere)". MORAVIA, La filosofia degli idéologues. Scienza dell'uomo e riflessione epistemologica tra Sette e Ottocento, in SANTATO, Letteratura italiana e cultura europea tra Illuminismo e Romanticismo, Atti del Convegno Internazionale di Studi, PadovaVenezia, 11-13 maggio 2000, pp. 77-78.

${ }^{20}$ CABANIS, Rapporti tra il fisico e il morale dell'uomo, traduzione integrale, p. 259. 
dal fatto che egli attribuisca la volontà alla vita animale, intendendo per essa, come solitamente avviene, solo l'arbitrio consapevole, che veramente si diparte dal cervello, dove però, come si è sopra mostrato, non è ancora un effettivo volere, bensì la mera ponderazione e il calcolo dei motivi, la cui conclusione o risultante si presenta infine come atto di volontà. Tutto ciò che io ascrivo alla volontà vera e propria, egli lo attribuisce alla vita organica, e tutto ciò che io intendo per intelletto è per lui la vita animale: questa ha per lui sede solo nel cervello e nelle sue appendici, quella invece in tutto il restante organismo. [...] ed è allora una gioia vedere come noi, come le due voci nel duetto, proseguiamo in reciproca armonia, sebbene qualcuno faccia intendere qualcosa di diverso. Quindi chi vuole capire me legga lui, e chi vuole capire lui più a fondo che non si sia capito egli stesso, legga me (W II, pp. 1493, 1495) ${ }^{21}$.

L'indagine bichatiana, a cui il filosofo rimanda, è esposta in particolare nelle Ricerche fisiologiche intorno alla vita e alla morte. Ci soffermiamo dunque sulle Recherches (1800), sul primo tomo dedicato alla vita, il secondo lo è alla morte, per esaminare in che termini Bichat descrive la vita animale e la vita organica, le loro funzioni, le loro differenze, e per comprendere i punti di contatto con la filosofia schopenhaueriana, in particolare con l'idea di unità corporea.

Bichat apre lo scritto ponendo la domanda intorno alla definizione della vita, che egli descrive come "l'unione delle funzioni che resistono alla morte ${ }^{22}$ ": "On cherche dans des considérations abstraites la définition de la vie; on la trouvera, je crois, dans cet aperçu général: la vie est l'ensemble des fonctions qui résistent à la mort ${ }^{23}$ ". La vita si contrappone alla morte non semplicemente nei termini della differenza, ma si pone in una relazione di antagonismo e resistenza nei suoi riguardi. Anche Schopenhauer tratteggia la vita similmente, come una "continua lotta per questa stessa esistenza, con la certezza di perderla in ultimo ${ }^{24}$ ” (W I, p. 611), come "un morire costantemente trattenuto, una morte sempre rimandata [... $]^{25 ”}$ (W I, p. 609).

Bichat prosegue spiegando che il resistere alla morte è la comune maniera di esistere dei corpi viventi, poiché tutto ciò che li circonda tende a distruggerli, a minarli in qualche misura. Anche i corpi inorganici esercitano gli uni sugli altri un'azione ininterrotta. Solo grazie all'interno principio di reazione, i corpi organici continuano a vivere. Tale principio coincide con quello stesso della vita, il quale, "sconosciuto nella sua natura, non può essere apprezzato se non se dietro i fenomeni da esso medesimo manifestati; il più generale dei quali consiste appunto in questa abituale alternativa d'azione per i corpi esterni, e di reazione per i corpi vivi; $[\ldots]^{26 "}$. La vita è dunque correlazione tra lo "sforzo"

\footnotetext{
${ }^{21}$ Oltre ai diversi richiami presenti negli scritti schopenhaueriani, si trovano riferimenti a Bichat e Cabanis anche negli scambi epistolari. Difatti, in un suo articolo, Paul Janet ricorda una lettera in cui emerge l'ammirazione schopenhaueriana per le ricerche dei due fisiologi: "Schopenhauer écrivait, en 1852, a son ami Frauenstädt: 'Il y a un certain V... qui se permet de traiter de superficiels les immortels écrits de Bichat et de Cabanis... Bichat n'a vecu que trente ans et toute l'Europe lettrée honore so nom et lit ses écrits... Sans doute, depuis lui, la physiologie a fait de progrès, mais non de manière à faire oublier Cabanis et Bichat... Je vous en prie, n'écrivez rien sur la physiologie dans son rapport à la psycologie sans avoir pris le suc et le sang de Cabanis et de Bichat.'” JANET, Schopenhauer et la physiologie française. Cabanis et Bichat, p. 35.

${ }^{22} \mathrm{BICHAT}$, Ricerche fisiologiche intorno alla vita e alla morte, p. 1.

${ }^{23} \mathrm{BICHAT}$, Recherches physiologiques sur la vie et la mort, p. 1.

${ }^{24}$ Proseguendo con la citazione, "La vita stessa è un mare pieno di scogli e di vortici, che l'uomo evita con la massima cautela e cura, benché sappia che, quand'anche riesca, con ogni sforzo ed arte, a scamparne, per ciò stesso si avvicinerà con ogni passo al più grande, al totale, all'inevitabile e irreparabile naufragio, anzi punterà direttamente su di esso, la morte. È questa la meta finale della faticosa traversata, e per lui è peggiore di tutti gli scogli che aveva scansati".

${ }^{25}$ Continuando con l'estratto, "ogni respiro respinge la morte sempre incalzante, con la quale noi in questo modo combattiamo ogni secondo, e poi ancora, a maggiori intervalli, con ogni pasto, con ogni sonno, con ogni riscaldarci ecc. Alla fine essa è destinata a vincere, giacché ad essa siamo commessi già dalla nascita, e soltanto essa scherza per un po' con la sua preda prima di ingoiarla. Frattanto noi continuiamo la nostra vita con grande partecipazione e molta cura, finché è possibile".

${ }^{26} \mathrm{BICHAT}$, Ricerche fisiologiche intorno alla vita e alla morte, p. 2.
} 
delle "potenze esterne" e quello della potenza interna, resistente, opponente, reagente. Tale è la "divisone generale" della vita.

Considerando la divisione della vita nel particolare, è possibile osservare che i vegetali hanno essenzialmente una vita interna, ossia si rapportano all'esterno soltanto per la nutrizione; gli animali invece hanno una vita esterna più complessa, che va ben al di là della sola nutrizione, abbracciando la sfera emotiva e cognitiva. Soffermandosi sulla vita animale, secondo Bichat si possono notare due ordini di funzioni: l'uno comprende i processi di assimilazione ed escrezione; l'altro riguarda il rapportarsi con l'esterno, attraverso gli organi di senso e il sistema nervoso, in una complessità di relazioni:

Io chiamo l'unione delle funzioni spettanti alla prima classe vita organica: siccome quella, che in un grado più o meno marcato è comune a tutti gli esseri vegetabili od animali; e siccome quella, che riconosce per sola condizione necessaria al suo esercizio la tessitura organica. Le funzioni riunite della seconda classe formano la vita animale, così chiamata perché essa è l'attributo esclusivo del regno animale ${ }^{27}$.

Si giunge a quello che per Schopenhauer è un fondamentale punto di contatto tra la fisiologia di Bichat e la sua filosofia: la vie organique corrisponde alla gradazione della volontà quale forza vitale, priva di coscienza, rispondente a stimoli; e la vie animale alla volontà animale, cosciente di se stessa e dell'altro, interagente con i motivi.

Vita organica e vita animale differiscono per le rispettive funzioni, in relazione alla forma, circa il modo di agire e di reagire, per il nascere, svilupparsi e perire. Bichat descrive puntualmente le loro specificità e il loro differenziarsi negli articoli che compongono le Recherches. Descrive anche il loro suggestionarsi, osservando in che modo la sfera intellettiva ed emotiva, riconducibile alla vita animale, influisce su quella organica e viceversa.

Una differenza tra le due forme di vita concerne la durata delle loro azioni: la vita organica è ininterrotta e infaticabile, come la volontà schopenhaueriana; la vita animale è invece intermittente e bisognosa di riposo, come l'intelletto schopenhaueriano ${ }^{28}$. Inoltre, è così necessario che le funzioni organiche, come la respirazione e la circolazione, siano costantemente mantenute, che, se per qualche motivo si sospendono in modo prolungato, possono porre fine alla vita; dato che sottolinea il carattere imprescindibile della vita organica. Diversamente, se una funzione animale, come l'udire o il vedere, si affievolisce, non causa la morte; dato che ne rivela il carattere secondario.

Anche l'influenza che l'abitudine esercita su una vita e sull'altra costituisce un tratto distintivo tra le due. Nei confronti della vie animale, secondo Bichat, l'abitudine agisce in modo diverso sul sentimento e sul giudizio, ottundendo il primo, perfezionando il secondo.

Al contrario, su un altro aspetto della vita animale, il carattere, l'abitudine non ha potere alcuno:

\footnotetext{
27 Ibidem, p. 4.

${ }^{28}$ Per il filosofo di Danzica la necessità di riposo propria della vie animale, quindi dell'Intellekt, a differenza dell'infaticabilità della vie organique, quindi del Wille inteso come forza vitale, costituisce un'ulteriore riprova della natura non originaria, e in tal senso non fondamentale, dell'intelletto. Soprattutto, tale necessità sembra ribadire che il principio vitale non risiede primariamente nel cervello ma nel cuore: "Niente dimostra più chiaramente la natura secondaria, dipendente, condizionata dell'intelletto che la sua intermittenza periodica. [...] Instancabile è solo il cuore, perché il suo battito e il circolo sanguigno non sono immediatamente condizionati dai nervi, ma sono appunto la manifestazione originaria della volontà. [...] Nel sonno, quando solo la vita vegetativa viene proseguita, la volontà opera soltanto secondo la sua natura originaria ed essenziale, non disturbata dall'esterno, senza detrazione della sua forza per l'attività del cervello e lo sforzo del conoscere, che è la funzione organica più difficile, ma per l'organismo è solo mezzo, non fine; quindi nel sonno tutta la forza della volontà è rivolta a conservare e, ove sia necessario, a migliorare l'organismo, ragion per cui ogni guarigione, tutte le crisi benefiche seguono nel sonno, in quanto la vis naturae medicatrix ha libero gioco solo quando è liberata dal fardello della funzione conoscitiva (W II, pp. 1455, 1457)". Come accennato in relazione a Cabanis, il bisogno di inattività, di distensione del pensiero è anche indicativo del suo avere una natura corporea, al pari di altre funzioni, che necessitano di riposo.
} 
Il carattere è, se posso così esprimermi, la fisionomia delle passioni: il temperamento è quella delle funzioni interne: ed essendo le une, e le altre invariabili, godendo cioè di una direzione, che non può essere mai dall'abitudine, e dall'esercizio alterata, riesce manifesto, come il temperamento, e il carattere devono essere del pari sottratti dall'impero dell'educazione. Può questa, è vero, moderare l'influenza del carattere, perfezionando il giudizio, e la riflessione, e fortificando la vita animale sì che questa resista alle impulsioni della vita organica, ma pretendere di snaturare col mezzo dell'abitudine il carattere, di raddolcire, ed esaltare le passioni, delle quali esso è l'espressione abituale, di restringere, e dilatare la loro sfera, sarebbe una intrapresa analoga a quella di un medico, il quale tentasse di innalzare, o di abbassare di qualche grado, e per tutta la vita, la forza di ordinaria contrazione del cuore nello stato di salute, di precipitare, o di rallentare abitualmente quel movimento che è naturale alle arterie, e necessario alla loro azione $\mathrm{ecc}^{29}$.

Questo passaggio rappresenta un ulteriore elemento della ricerca bichatiana che Schopenhauer condivide con ammirazione, collimando con la sua idea di carattere immodificabile, nella sua intima essenza, esposta soprattutto nello scritto La libertà del volere umano.

Intorno a tale questione, così commenta Schopenhauer:

Ma ciò che nel corso dei pensieri di Bichat è più ammirevole e per me più allietante, è che questo grande anatomista giunge, attraverso le sue considerazioni puramente fisiologiche, fino a spiegare l'immutabilità del carattere morale col fatto che solo la vita animale, cioè la funzione cerebrale, è soggetta all'influsso dell'educazione, dell'esercizio, della formazione e dell'abitudine, mentre il carattere morale appartiene alla vita organica non modificabile dal di fuori, ossia a quella di tutte le altre parti del corpo (W II, p. 1497).

Proseguendo con le differenze tra la vita organica e la vita animale, un'altra concerne l'azione delle passioni e del pensiero sulle due vite; azione che rivela il carattere unitario del corpo umano, al di là delle distinzioni.

[...] Egli è certamente meraviglioso, che le passioni, le quali fanno essenzialmente parte delle nostre relazioni cogli oggetti che ci circondano; le quali ad ogni istante modificano queste relazioni, senza di che la vita animale non sarebbe che una fredda serie di fenomeni intellettuali; le quali animano, aggrandiscono, esaltano continuamente tutti i fenomeni di questa vita; egli è, dico, meraviglioso, che le passioni non abbiano mai né il loro termine né la loro origine nei vari organi di lei; e che al contrario siano costantemente da esse affetti gli organi destinati alle funzioni interne: e che queste siano pure da questi variamente determinate secondo il vario loro stato ${ }^{30}$.

Ebbene, non solo per Schopenhauer e Cabanis, ma anche per Bichat, le passioni causano alterazioni nella vita organica.

L'ira accelera la circolazione e accresce il ritmo cardiaco, influenzando la forza e la rapidità del moto sanguigno. Anche la gioia è in grado di cambiarlo sensibilmente, velocizzandolo e spingendolo verso l'organo cutaneo. Il timore influisce in modo opposto, indebolendo il sistema vascolare, e così, non pervenendo sangue ai vasi capillari, si produce il pallore lungo tutto il corpo ma soprattutto sul volto. Le passioni mutano anche il respiro, si pensi all'oppressione che irrompe per un profondo dolore, provocando un'alterazione

${ }^{29}$ BICHAT, Ricerche fisiologiche intorno alla vita e alla morte, p. 157.

30 Ibidem, pp. 56-57. 
respiratoria. Le emozioni condizionano gli organi della digestione, potendo causare conati di vomito, contrazioni prolungate, disequilibrio funzionale. Anche il funzionamento degli organi secretori è modificato dai moti dell'animo: uno spavento può sospendere il corso della bile, determinando l'itterizia; un eccesso di collera può disporre a una febbre biliosa; contentezza e sofferenza possono provocare una copiosa lacrimazione; l'ipocondria può affettare il pancreas ${ }^{31}$.

Inoltre, espressioni quali l'ardere dall'invidia, l'essere consunto dalla tristezza, l'essere corroso dai rimorsi ben indicano la coappartenenza di psichico e somatico. Il furore che circola nelle vene o la gioia che fa palpitanti $i$ visceri sono proposizioni che riflettono i moti del corpo. "Non sono", scrive Bichat, "già metafore impiegate dai poeti, ma espressioni di ciò che trovasi realmente in natura ${ }^{32}$ ". Similmente, gli epiteti attribuiti alla testa e al cuore indicano le rispettive specificità: una testa ben formata o quadrata suggerisce la perfezione dell'intelletto; un buon cuore, un cuore sensibile allude a un bel carattere. Sono tratti sui quali anche Schopenhauer si sofferma, per sottolineare come le prerogative dell'animus siano legate alla volontà, mentre quelle della mens all'intelletto. Tutto ciò che riguarda la volontà, dal carattere a un'emozione a una qualità morale, è attribuito al cuore, e tutto ciò che riguarda l'intelletto, e dunque la conoscenza, in un senso molto ampio, è attribuito all'intelletto:

Come sono indovinate e perspicue le espressioni quotidiane 'testa' e 'cuore'! [...] Cuore significa sempre la volontà e tutto quanto le appartiene:

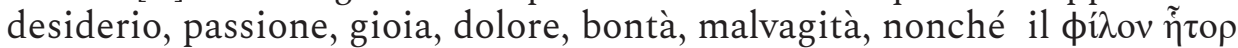
di Omero. 'Gli viene dal cuore', 'gli si spezza il cuore', 'gli batte il cuore per la gioia', 'non ne ha il cuore', 'un colpo al cuore', 'che spacca il cuore', 'che tocca il cuore', 'che rasserena il cuore', 'cordiale', 'di buon cuore', 'senza cuore', 'duro di cuore', 'coraggioso', 'di cuore vile'. [...] La testa, al contrario, indica la rappresentazione, la conoscenza e tutto quanto le appartiene: 'un uomo di testa', 'una testa fina', 'una testa assennata', 'perdere la testa', 'tenere alta la testa'. [...] Testa e cuore costituiscono la totalità dell'uomo, come la volontà e la rappresentazione: una volta che si siano elogiati testa e cuore, non v'è più nulla da criticare (HN III, p. 107).

Oltre all'influenza delle passioni e dei pensieri sull'organismo, occorre non trascurare l'influsso contrario, quello del corpo sui pensieri e l'emotività:

Oltre di questo noi dobbiamo ancora osservare che non solo le passioni portano sulle funzioni organiche la loro influenza, dirigendo in modo speciale le loro impressioni ai visceri di queste funzioni, ma il vario stato, le varie lesioni, e le variazioni di forze di questi visceri concorrono, in modo assai evidente, alla produzione delle passioni; il qual fatto viene incontrastabilmente provato dai rapporti, che uniscono le passioni stesse coi temperamenti, colle età ecc. ${ }^{33}$

Ad esempio, un individuo dall'apparato polmonare pronunciato, con un sistema circolatorio molto energico, "assai sanguigno", tende a mostrare un certo impeto che lo dispone soprattutto al coraggio, al trasporto, alla collera. Al contrario, un individuo in cui predominano le funzioni dei linfatici tende ad avere un temperamento più pacato. Un individuo con un sistema bilioso deciso sembra essere predisposto a sentimenti malevoli, come l'invidia e l'astio.

Ora, sebbene per Bichat le passioni influenzino con maggiore evidenza e forza le funzioni della vita organica, questo non significa che non modificano le funzioni della

\footnotetext{
${ }^{31}$ A riguardo, cfr. BICHAT, Ricerche fisiologiche intorno alla vita e alla morte, pp. 57-60.

32 Ibidem, p. 62.

${ }^{33}$ Ibidem, p. 63.
} 
vita animale. Tutt'altro. Può accadere, a causa di una violenta passione, che il sistema nervoso non sia in grado di coordinare i movimenti come solitamente accade ${ }^{34}$. Anche gli organi di senso possono alterarsi, la vista può annebbiarsi e le orecchie possono ronzare. Un uomo in uno stato collerico, avendo la circolazione più accelerata, ha una considerevole energia cerebrale. Pertanto, il cervello potrebbe determinare i muscoli sottoposti alla sua influenza ad agire con veemenza, a tal punto che i movimenti diventano involontari, simili a spasmi, e probabilmente le espressioni linguistiche hanno affini andamenti. Al contrario, in uno stato di timore, poiché perviene al cervello un minore afflusso sanguigno, i movimenti da esso coordinati ne sono indeboliti, e le parole diventano balbettii. Una passione può dunque turbare anche il linguaggio, che è un aspetto della vita animale.

Con Cabanis si è visto in che misura i pensieri sono influenzati dagli stati d'animo, a tal punto che egli definisce le "impressioni" essere in noi e non nelle cose. Anche per Schopenhauer il pensiero non è affatto slegato dall'elemento volitivo, potendo essere turbato, rallegrato, confuso da un sentimento: "L'intelletto è come la superficie specchiante dell'acqua, e questa stessa è come la volontà, il cui agitarsi quindi fa venir subito meno la purezza di quello specchio e la nitidezza delle sue immagini. L’organismo è la volontà stessa, è volontà incarnata in un corpo [...] (W II, p. 1411) 35 ”.

Schopenhauer paragona la volontà anche alle radici di un albero, giacché entrambe elemento essenziale alla vita, a partire da cui sorgono e si sviluppano le altre parti del vivente. L'intelletto è considerato analogo alla corona, il cui germogliare non è slegato dall'attività delle radici. Se queste periscono, la chioma sfiorisce. "La radice rappresenta la volontà, la corona l'intelletto, e il punto di indifferenza di entrambi, il rizoma, sarebbe l'Io che, come terminale comune, appartiene a entrambi” (W II, p. 1385). Come le radici, la corona e il rizoma sono parti che compongono la struttura dell'albero, e sono relate le une alle altre, così l'elemento emotivo, intellettivo e organico costituiscono quel fenomeno unitario che il corpo umano è.

Un pensiero e una passione si rivelano come corporeità, un'attività organica può mutare uno stato d'animo. Metafisica e fisiologia si incontrano.

\section{Riferimenti bibliografici}

BICHAT, Xavier. Recherches physiologiques sur la vie et la mort. Paris: chez Brosson-Gabon, 1802 (deuxième edition).

\footnotetext{
${ }^{34}$ A riguardo, cfr. BICHAT, Ricerche fisiologiche intorno alla vita e alla morte, pp. 66-72. Emblematico dell'influsso di una passione, tale da disturbare l'azione del sistema nervoso, è il seguente caso che Bichat riporta: "Sotto le passioni veementi, l'affezione assai viva degli organi interni eccita con tal impeto i movimenti simpatici dei muscoli, che viene assolutamente tolta ogni azione su di essi dal cervello esercitata; ma cessata poi questa prima impressione, si restituisce il modo ordinario di locomozione. Perché riesca ciò chiaro, osserviamo un uomo, il quale in una conversazione riceva col mezzo di una lettera una notizia, che a lui prema di nascondere; egli ad un tratto impallidisce, gli si corruga la fronte, o si anima la sua fisionomia a seconda della passione in esso eccitata; questi fenomeni simpatici sono nati da qualche viscere addominale repentinamente affetto da tale passione; e che perciò appartengono alla vita organica. Ben presto però quest'uomo tenta di riaversi; e la sua fronte si rasserena: rinasce il primiero suo colorito, e la sua fisionomia torna al pristino stato, benché l'interno sentimento ancora sussista; in tal caso adunque al moto volontario vince il simpatico; l'azione del cervello supera quella dello stomaco, del fegato ecc. e la vita animale riprende il suo impero". Ibidem, pp. 70-71.

35 Inoltre, il filosofo osserva che la necessità, in un senso ampio, può donare capacità anche agli uomini, e agli altri animali, meno avveduti: "Un motivo che operi con forza, come un desiderio nostalgico, una necessità pressante, potenzia talvolta l'intelletto fino a un grado di cui prima non lo avevamo mai ritenuto capace. Circostanze difficili, che ci impongono la necessità di fare certe cose, sviluppano in noi talenti del tutto nuovi, i cui germi ci erano rimasti celati e per cui credevamo di non avere nessuna attitudine. L'intelligenza dell'uomo più ottuso si fa acuta, quando si tratti di oggetti molto importanti del suo volere [...]. Perfino l'intelligenza degli animali viene notevolmente accresciuta dalla necessità, sicché essi fanno in casi difficili cose che ci fanno stupire: per esempio quasi tutti calcolano che sia più sicuro non fuggire, quando credono di non essere visti; [...]" (W II, p. 1419).
} 
BICHAT, Xavier. Ricerche fisiologiche intorno alla vita e alla morte. Prima traduzione italiana fatta sulla terza edizione francese. Pavia: nella stamperia Fusi e Comp. ${ }^{\circ}, 1823$.

CABANIS, Pierre. Rapporti tra il fisico e il morale dell'uomo. Prima versione italiana, eseguita sopra la terza edizione francese, preceduta per la prima volta da una tavola analitica del Conte Destutt de Tracy e seguita da una tavola alfabetica del Signor Sue, 1820; Rapporti tra il fisico e il morale dell'uomo. Traduzione parziale a cura di Sergio Moravia. Roma-Bari: Editori Laterza, 1973.

CABANIS, Pierre. Coup d'œil sur les révolutions et sur la réforme de la médecine. Paris: Crapart, Caille et Ravier, 1804.

DOBO, Nicolas, ROLE, André. Bichat. La vie fulgurante d'un génie. Paris: Perrin, 1989.

DI PALO, Lucia. Les Recherches physiologiques sur la vie et la mort di Fraçois Xavier Bichat. Un lessico fisiologico. Bari: Cacucci editore, 2005.

GRIGENTI, Fabio. Natura e rappresentazione. Genesi e struttura della natura in Arthur Schopenhauer. Istituto Italiano per gli Studi Filosofici, Napoli: La città del sole, 2000.

JANET, Paul. Schopenhauer et la physiologie française. Cabanis et Bichat. Revue des deux mondes, Paris, Mai 1880, pp. 35-59.

KLOPPE, Wolfgang. Die Bestätigung der Philosophie Arthur Schopenhauers (1788-1860) durch das Werk des Physiologen Xavier Bichat (1771-1802). Eine Begegnung von Physik und Metaphysik. Medizinische Monatsschrift, 22, 1968, pp. 306-312.

LEFRANC, Jean. Schopenhauer lecteur de Cabanis. Revue de Métaphysique et de Morale, v. 88, n. 4, 1983, pp. 549-557.

MANDELBAUM, Maurice. The Physiological Orientation of Schopenhauer's Epistemology, in FOX, Michael (ed. by). Schopenhauer. His Philosophical Achievement. Totowa (N.J.): Barnes \& Noble Books, 1980, pp. 50-67.

MORAVIA, Sergio. Introduzione, in CABANIS, Pierre-Jean-Georges. Rapporti tra il fisico e il morale dell'uomo. Roma-Bari: editori Laterza, 1973, pp. VII-XXXIX.

MORAVIA, Sergio. Il pensiero degli idéologues. Scienza e filosofia in Francia (1780-1815). Firenze: La nuova Italia, 1974.

MORAVIA, Sergio. La filosofia degli idéologues. Scienza dell'uomo e riflessione epistemologica tra Sette e Ottocento, in SANTATO, Guido (a cura di). Letteratura italiana e cultura europea tra Illuminismo e Romanticismo, Atti del Convegno Internazionale di Studi, Padova-Venezia, 11-13 maggio 2000. Genève: Librairie Droz S.A., 2003, pp. 65-79.

SCHOPENHAUER, Arthur. La vista e i colori e carteggio con Goethe. A cura di Mazzino Montinari. Milano: Abscondita, 2002.

SCHOPENHAUER, Arthur. Il mondo come volontà e rappresentazione, volume I e II. Introduzione e traduzione di Sossio Giametta, bibliografia e indici di Vincenzo Cicero. Milano: Bompiani, 2010.

SCHOPENHAUER, Arthur. Sulla volontà nella natura. A cura di Sossio Giametta. Milano: BUR, 2010.

SCHOPENHAUER, Arthur. La libertà del volere umano. Introduzione di Cesare Vasoli, traduzione di Ervino Pocar. Roma-Bari: Laterza, 1970.

SCHOPENHAUER, Arthur. Parerga e Paralipomena. Scritti filosofici minori. Tomo primo a cura di Giorgio Colli, tomo secondo a cura di Mario Carpitella, tr. it. di Mazzino Montinari e Eva Kühn Amendola. Milano: Adelphi, 2007.

SCHOPENHAUER, Arthur. Scritti Postumi, volume I, I manoscritti giovanili (1804-1818). Testo stabilito da Arthur Hübscher, edizione italiana diretta da Franco Volpi, volume a cura di Sandro Barbera. Milano: Adelphi, 1996.

SCHOPENHAUER, Arthur. Scritti Postumi, volume III, I manoscritti berlinesi (1818-1830). Testo stabilito da Arthur Hübscher, edizione italiana diretta da Franco Volpi, volume a cura di Giovanni Gurisatti. Milano: Adelphi, 2004. 
SCHOPENHAUER, Arthur. Lezioni filosofiche, II, Metafisica della natura. A cura di Ignazio Volpicelli. Roma-Bari: Laterza, 2007.

SCHMIDT, Alfred. Physiologie und Transzendentalphilosophie bei Schopenhauer. SchopenhauerJahrbuch, 1989, pp. 43-53.

SCHUBBE, Daniel, SØREN, R. Fauth. Schopenhauer und Goethe. Biographische und philosophische Perspektiven. Hamburg: Felix Meiner Verlag, 2016.

SEGALA, Marco. Fisiologia e metafisica in Schopenhauer. Rivista di filosofia, v. LXXXV, n. 1, 1994, pp. 35-66.

SEGALA, Marco. Schopenhauer, Goethe e l'ottica sperimentale. Rivista di Filosofia, v. XCVI, n. 2, 2005, pp. 217-231.

SEGALA, Marco. Schopenhauer, la filosofia, le scienze. Pisa: Edizioni della Normale, 2009.

SEGALA, Marco. The Role of Physiology in Schopenhauer's Metaphysics of Nature. SchopenhauerJahrbuch, 2012, pp. 327-334.

VOLPICELLI, Ignazio. A. Schopenhauer. La natura vivente e le sue forme. Settimo milanese: Marzorati editore, 1988.

TAKAHASHI, Yoichiro. Lebensphänomenologie durch Physiologie in der mittleren und späten Periode Schopenhauers. Schopenhauer-Jahrbuch, 2012, pp. 163-174. 